\title{
Direct Analysis of Gene Synthesis Reactions Using
}

\section{Solid-State Nanopores}

\author{
Spencer Carson ${ }^{1,2}$, Scott T. Wick ${ }^{3}$,Peter A. Carr ${ }^{3}$, Meni Wanunu ${ }^{1,2^{*}}$, Carlos A. Aguilar ${ }^{3 *}$
}

${ }^{1}$ Department of Physics, Northeastern University, Boston, MA, USA, ${ }^{2}$ Department of Chemistry and Chemical Biology, Northeastern University, Boston, MA, USA, ${ }^{3}$ Massachusetts Institute of Technology Lincoln Laboratory, 244 Wood St., Lexington, MA 02420.

*wanunu@neu.edu, *carlos.aguilar@11.mit.edu

Supplemental Information 
a)

5' -GGAGGCTTCTGTGTCTTGAAGAAGGACCAGGGAACGTATTTGCCTTGTCTGGGAAATCGTGTGAAATCTG-3'
3' -CCTCCGAAGACACAGAACTTCTTCCTGGTCCCTTGCATAAACGGAACAGACCCTTTAGCACACTTTAGAC-5'

70+71bp Mismatch

5' -GGAGGCTTCTGTGTCTTGAAGAAGGACCAGGGAACGTATTT-GCCTTGTCTGGGAAATCGTGTGAAATCTG-3'

3' - CCTCCGAAGACACAGAACTTCTTCCTGGTCCCTTGACATAAACGGAACAGACCCT'TAGCACACTTTAGAC -5'

b)

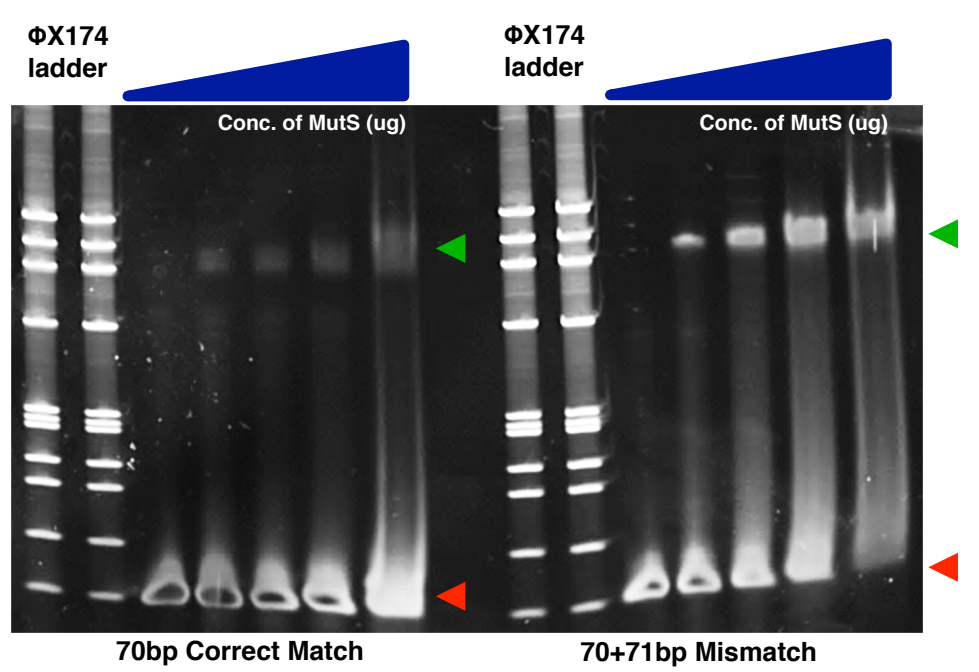

Supplemental Figure 1. a) Sequences of 70nt "correctly matched" oligos and 70nt/71nt "mismatch" duplex. The single-base mismatch is highlighted in yellow and located $35 \mathrm{bp}$ from either end. b) Gel shift electrophoresis assay of the correctly matched and mismatch products incubated with various amounts of MutS. The red triangle indicates the migration of the $70 \mathrm{bp}$ and $70 / 71 \mathrm{bp}$ product bands and the green triangle indicates the migration of the MutS + dsDNA complex. As the concentration of MutS was increased, the intensity of the complex band also increased, indicating a significant fraction of the mismatch products were bound to the MutS. In contrast, little to no binding is observed for the correctly matched products. 


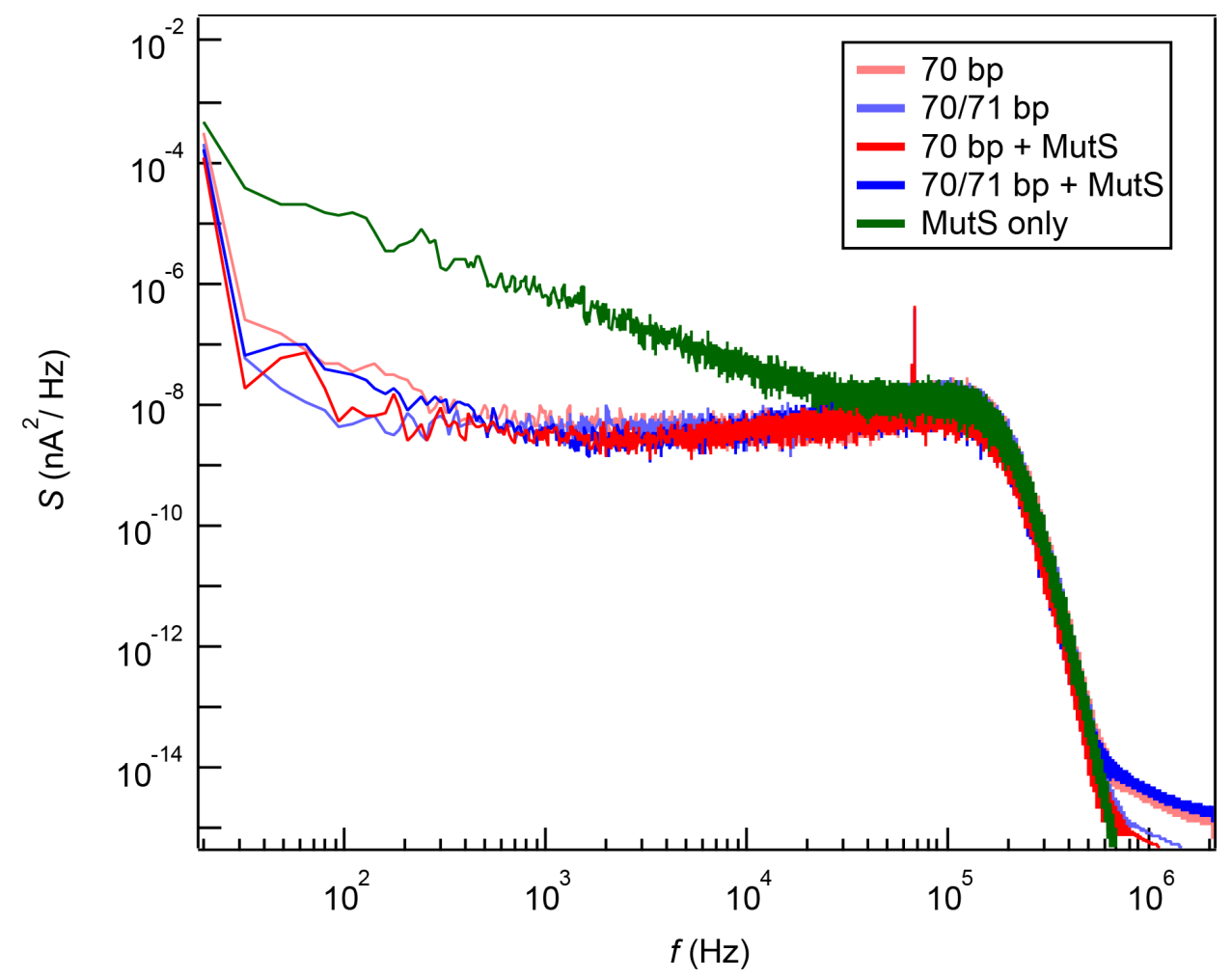

Supplemental Figure 2. Power spectral density (PSD) plots based on $500 \mathrm{~ms}$ of open pore current data for each sample of $70 \mathrm{bp}$ (pink), 70/71 bp (light blue), $70 \mathrm{bp}+$ MutS (red), 70/71 bp + MutS (blue), and MutS only (green) at $200 \mathrm{mV}$ in small silicon nitride nanopores. In the case of DNA incubated with MutS, each PSD had a notable spike at a frequency of $\sim 70,000 \mathrm{~Hz}$ not present in the experiment without MutS, which is due to environmental noise from electronics. We also produced a PSD plot for when the MutS protein clogs a small nanopore in the absence of DNA (green), which was accompanied by a $61 \%$ reduction in the pore conductance (i.e., $720 \mathrm{pA}$ to $280 \mathrm{pA}$ ). This PSD has noticeably higher noise at all frequencies, which indicates that MutS is not stuck to the pore in our DNA/MutS experiments. 
a)

I. Overhang

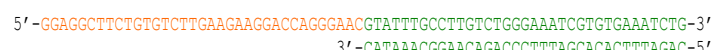

II. Holliday Junction

5' 3' -CACTCCTGTCACATCATAATCGTT'TGCTATTCAGGCATAAACGGAACAGACCCT'TTAGCACACTTTAGAC-5'

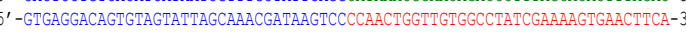
3'- -CCTCCGABGACACAGAACTICTTCCTGGTCCCTIGGGTTGACCAACACCGGATAGCTTTTCACTTGABGT-5

\section{Flap}

5' -GGAGGCTTCTGTGTCTTGAAGAAGGACCAGGGAACGTATTTGCCTTGTCTGGGAAATGTGTGAAATCTG-3' $3^{\prime}$ - CATAAACGGAACAGACCCTITAGCACACTTTAGAC-5'

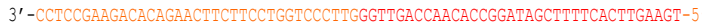

c)
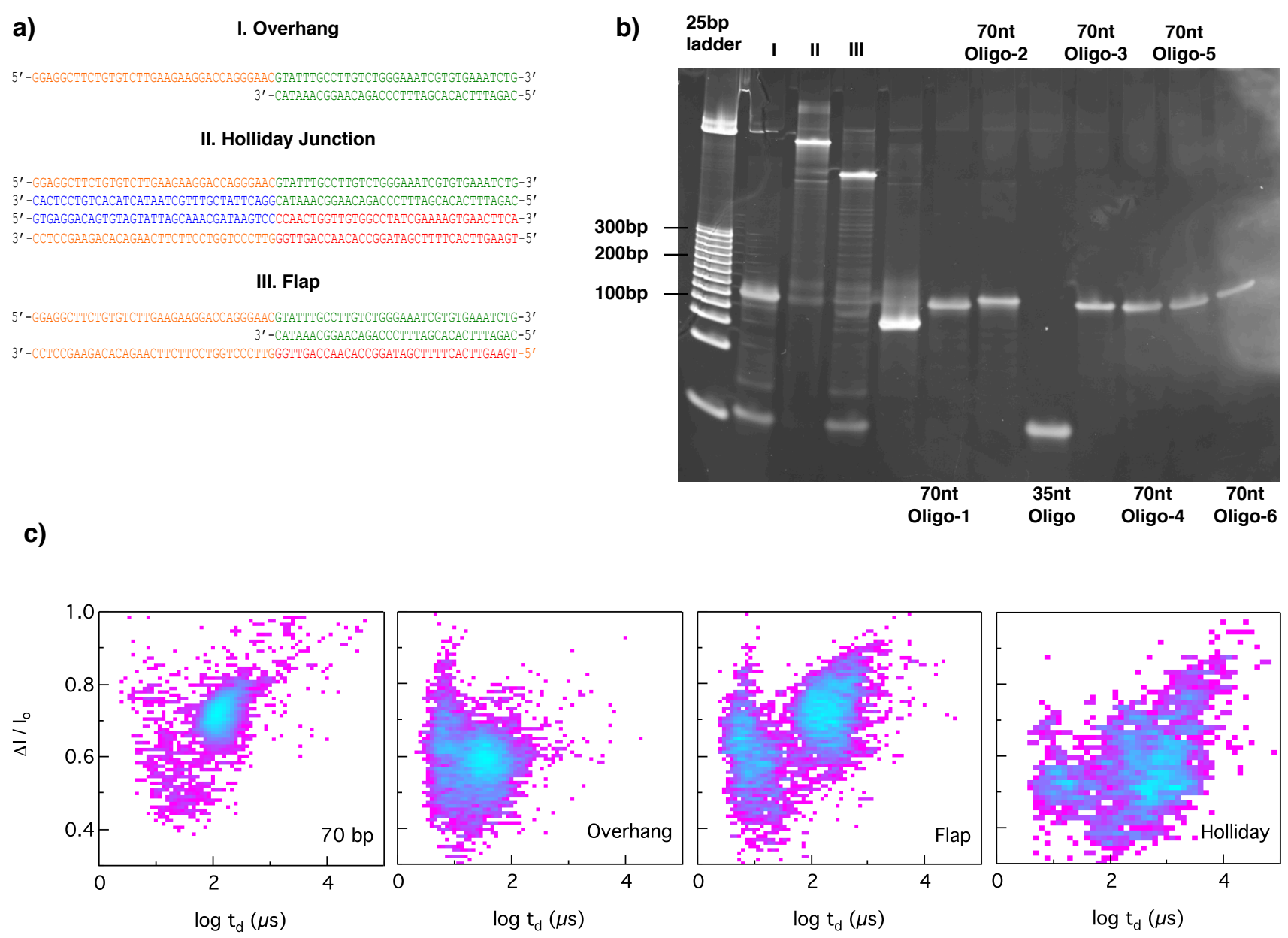

Supplemental Figure 3. a) Sequences of 35 nt and 70 nt oligos used to create different types of defective structures (overhang, flap and Holliday junction). Seven oligos were used and bases paired with other oligos are color-coded. b) Gel shift electrophoresis assay of the different assembled products and oligos. c) Heat map contour plots of $\Delta I / I_{O}$ vs $t_{d}$ for different assembled products translocating through a nanopore. 


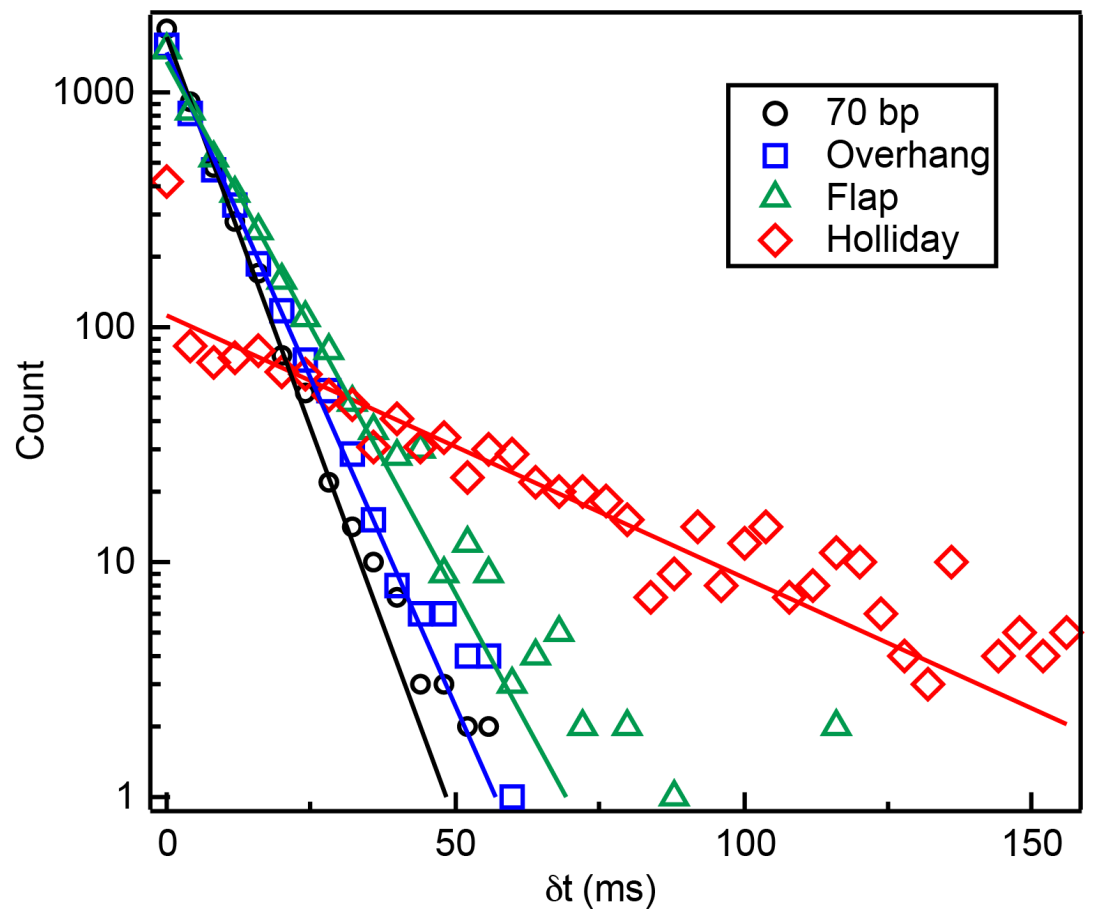

Supplemental Figure 4. Inter-event time histograms of gene synthesis mis-assemblies. Fitting interevent time histograms to single exponential functions allows the extraction of capture rate $R_{C}$ for each sample as displayed in the table. All capture rates were normalized for sample concentration as measured by spectrophotometer. The Holliday junction was found to have a capture rate an order of magnitude lower than the $70 \mathrm{bp}$ sample, and the overhang and flap molecules had comparable $R_{C}$. 
a)

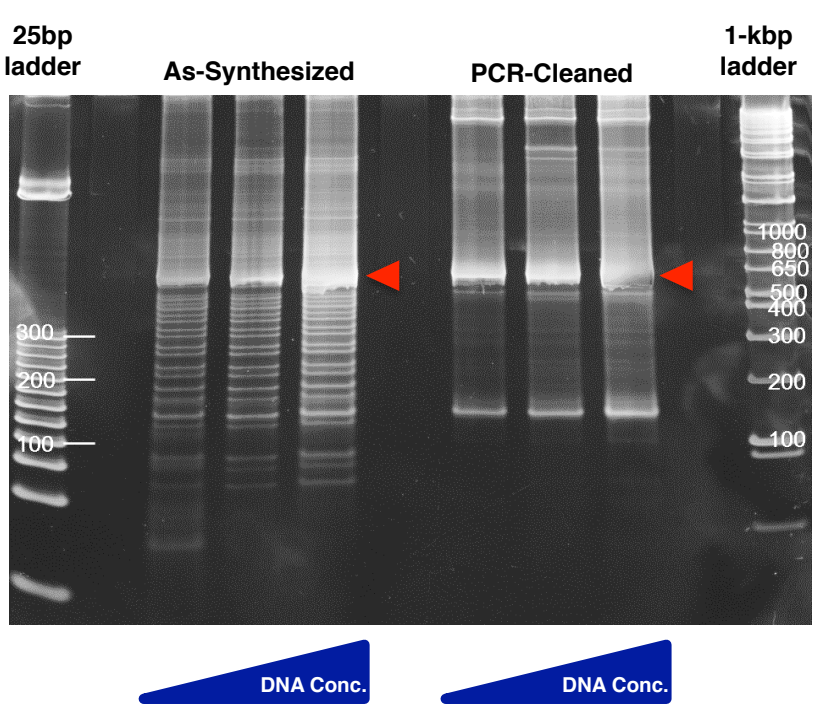

b)

\begin{tabular}{|c|c|}
\hline HIVprNOGAP-t1 & ATGAATCGGCCAACGTCCGGCGTAGAGGCGAAATTAATACGACTCACTATAGGGAG \\
\hline HIVprNOGAP-b2 & ATTTCTAGAGGGAAACCGTTGTGGTCTCCCTATAGTGAGTCGTATTAATTTCGC \\
\hline HIVprNOGAP-t3 & ACCACAACGGTTTCCCTCTAGAAATAATTTTGTTTAACTTTAAGAAGGAGATATACCATG \\
\hline HIVprNOGAP-b4 & AGGGTAACCTGCGGACCAGACATGGTATATCTCCTTCTTAAAGTTAAACAAAATT \\
\hline HIVprNOGAP-t5 & TCTGGTCCGCAGGTTACCCTGTGGCAGCGTCCGCTGG \\
\hline HIVprNOGAP-b6 & AGCTGACCACCAATTTTGATGGTAACCAGCGGACGCTGCCAC \\
\hline HIVprNOGAP-t7 & TTACCATCAAAATTGGTGGTCAGCTCAAAGAAGCGCTGCTGGACAC \\
\hline HIVprNOGAP-b8 & CGGTGTCGTCCGCACCCGTGTCCAGCAGCGCTTCTTTG \\
\hline HIVprNOGAP-t9 & GGGTGCGGACGACACCGTTCTGGAAGAGATGTCTCTGCCG \\
\hline HIVprNOGAP-b10 & ATCATTTTCGGCTTCCAACGACCCGGCAGAGACATCTCTTCCAGAA \\
\hline HIVprNOGAP-t11 & GGTCGTTGGAAGCCGAAAATGATCGGCGGTATCGGTGGCTTC \\
\hline HIVprNOGAP-b12 & TCTGGTCGTACTGACGAACTTTGATGAAGCCACCGATACCGCCG \\
\hline HIVprNOGAP-t13 & ATCAAAGTTCGTCAGTACGACCAGATCCTGATCGAAATCTGCGGCC \\
\hline HIVprNOGAP-b14 & AGTACCGTACCGATCGCTTTGTGGCCGCAGATTTCGATCAGGA \\
\hline HIVprNOGAP-t15 & ACAAAGCGATCGGTACGGTACTGGTTGGTCCGACCCCGGT \\
\hline HIVprNOGAP-b16 & GGAGGTTGCGACCGATGATGTTAACCGGGGTCGGACCAACC \\
\hline HIVprNOGAP-t17 & TAACATCATCGGTCGCAACCTCCTCACCCAGATCGGTTGCACC \\
\hline HIVprNOGAP-b18 & TGGGCAACAACCGAAGTTCAGGGTGCAACCGATCTGGGTGA \\
\hline HIVprNOGAP-t19 & CTGAACTTCGGTTGTTGCCCAGGTTGCTGTTAATAACTAGCATAA \\
\hline HIVprNOGAP-b20 & GACCCGTTTAGAGGCCCCAAGGGGTTATGCTAGTTATTAACAGCAACC \\
\hline HIVprNOGAP-t21 & CTTGGGGCCTCTAAACGGGTCTTGAGGGGTTTTTTTGCTGAAAGGAG \\
\hline HIVprNOGAP-b22 & CCGCCTCTCCCCGCGAGTTCCTCCTTTCAGCAAAAAACCCCTCAA \\
\hline
\end{tabular}

Supplemental Figure 5. a) Gel electrophoresis assay of the as-synthesized HIVPr synthetic gene and PCR-cleaned HIVPr synthetic gene. The red triangle shows the migration of the correctly assembled product band. The gel shows both correctly assembled products (524 bp) and incorrectly assembled products (such as oligos, intermediate assemblies of shorter length, as well as longer fragments) are present before and after cleanup with PCR, though the fraction of incomplete assemblies was markedly reduced after PCR cleanup. b) List of 22 construction oligos with names and sequences used to construct the HIVPr gene. 